Proclamation d'une nouvelle matérialité pour l'éthique dans les entreprises - forme d'organisation inédite du travail ou réaménagement des effets de la communication symbolique?

\title{
Catherine Loneux
}

\section{OpenEdition}

Édition électronique

URL : http://journals.openedition.org/communicationorganisation/2493

DOI : 10.4000/communicationorganisation.2493

ISSN : 1775-3546

Éditeur

Presses universitaires de Bordeaux

Édition imprimée

Date de publication : 1 mai 2001

ISSN : 1168-5549

\section{Référence électronique}

Catherine Loneux, «Proclamation d'une nouvelle matérialité pour l'éthique dans les entreprises forme d'organisation inédite du travail ou réaménagement des effets de la communication symbolique? », Communication et organisation [En ligne], 19 | 2001, mis en ligne le 27 mars 2012, consulté le 19 avril 2019. URL : http://journals.openedition.org/communicationorganisation/2493 ; DOI : 10.4000/communicationorganisation.2493

Ce document a été généré automatiquement le 19 avril 2019.

(c) Presses universitaires de Bordeaux 


\title{
Proclamation d'une nouvelle matérialité pour l'éthique dans les entreprises - forme d'organisation inédite du travail ou réaménagement des effets de la communication symbolique?
}

\author{
Catherine Loneux
}

Pendant plusieurs années (quatre-vingt surtout), on a vu se développer massivement des interrogations sur la place centrale des codes éthiques dans les entreprises, appelées à évoluer dans un contexte socio-économique changeant, où il fallait se préserver des tentatives de corruption et des conflits d'intérêts. Les discours de cette époque, simples ingrédients des politiques de communication efficaces et stratégiques, n'avaient pas eu d'effets majeurs autres que symboliques à l'intérieur des entreprises. Ils ont par la suite été taxés, par ceux-là même qui les prononçaient, de phénomènes de mode, appelés de leur vœu à être rapidement remplacés par une véritable volonté d'application concrète des principes éthiques.

2 Aujourd'hui, la stratégie affichée des directions d'entreprises consiste à vouloir intégrer l'éthique à des objectifs matériels, touchant au contrôle et à la recomposition des organisations (nouveaux métiers, nouvelles fonctionnalités). On se situe là dans les grandes entreprises d'origine plutôt anglo-saxonne qui possèdent des filiales en France et en Europe, et qui sont les plus touchées par l'apparition des nouveaux métiers de « déontologues ", dont la mission consiste à jouer un rôle d'experts ( $90 \%$ des grandes entreprises américaines ont une politique éthique formalisée (Mercier, p. 10. 1999). Nous souhaitons faire le point sur les motivations des dirigeants d'entreprise du XXI ${ }^{e}$ siècle : les effets de matérialité de l'éthique qu'ils proclament par leurs discours sont bien réels, mais nous formulons l'hypothèse que la mission du déontologue a essentiellement pour objet 
d'innover en matière de stratégies de communication, afin d'orienter celles-ci vers une démarche de "gestion du risque de réputation ».

3 A cet égard, la " gestion grise » pratiquée par les experts affiche des effets de matérialité (la présence réelle du déontologue, des codes, des chartes éthiques, l'élargissement des cibles touchées par son discours éthique), autrement dit des objectifs instrumentaux, dissimulés derrière de l'immatérialité (les dispositifs de chartes éthiques servent encore à penser, connaitre, croire, pour transmettre, inculquer, et avoir en commun et renforcer des représentations, agir sur la subjectivité du salarié).

Vouloir impliquer les déontologues dans la stratégie globale de l'entreprise, installer un tel espace social dans une organisation, tout cela n'est possible que grâce à la communication. Si ces métiers sont apparus, c'est uniquement avec la communication qu'ils subsistent, même si cela reste parfois caché. L'expertise vit, et s'installe grâce à la communication, et nous sommes là face à une situation où une dimension matérielle certes nouvelle ne peut se passer d'une dimension immatérielle pour exister. Il y a toujours une perte de sens dans la transmission, mais elle est largement compensée par les discours agrémentant les codes.

5 Sur le plan de la méthode, nous inscrivons ce travail dans la lignée des études et observations anthroposociologiques sur l'influence des modes de management dans la structuration des entreprises et des groupes sociaux qui la composent (articulation des logiques d'acteurs et de la communication). Nous rejoindrons plus précisément, au plan théorique, la critique de l'expertise et sa capacité de rationalisation des rapports et des usages sociaux à l'intérieur des systèmes de production et des entreprises.

Deux axes d'étude seront proposés :

7 1) L'observation d'une volonté de dynamisme et de recomposition concrète des professionnalismes soutenue par des productions symboliques autour des codes, des chartes éthiques et des écrits professionnels (I).

8 2) L'interrogation sur la réalité de ces effets matériels annoncés : par le biais de discours sur l'apparition de "nouveaux" métiers, ne se trouve-t-on pas plutôt face à des préoccupations d'image, à une forme réinventée de la communication et de la médiatisation des entreprises, en leur sein et dans l'espace public plus généralement, par la structuration et la régulation de ses propres circuits d'acteurs (II)?

\section{L'expertise éthique du déontologue : une forme de matérialité reliée à la communication}

9 La pratique du déontologue est reliée à son habitude de dialogue dans l'entreprise avec les responsables opérationnels, de qui il peut obtenir une coopération dans l'application et le respect de certaines procédures (contrôle financier, etc.). Un élément immatériel, culturel, se dissimule derrière la matérialité de cette profession. La définition de l'expertise éthique nous intéresse surtout pour sa dimension instituante, qui renvoie aux interactions qu'elle vise avec le public, et aux discours qu'elle propose.

10 Nous appuierons notre propos sur l'examen d'un corpus de traces écrites, essentiellement, d'articles de presse généraliste, spécialisée, mais aussi de témoignages de professionnels appartenant au Cercle Ethique des Affaires, ou au Cercle Européen des Déontologues. 


\section{Apparition de nouveaux métiers}

11 Les fonctions réservées à l'expertise présentent des contours flous. Elles peuvent être globalement séparées en deux catégories, comme proposé par Bernard Floris (Floris, 1994). La première renvoie à des situations de litige entre des parties adverses qui nécessitent la nomination d'un médiateur. La deuxième renvoie plutôt à la recherche de solutions à des dysfonctionnements organisationnels ou plus simplement à des questions d'organisation, et là l'expert sera convoqué pour sa capacité à avoir une vision globale de l'organisation, afin d'exercer un rôle de spécialiste éclairé et de proposer des solutions. Il disposera pour cela de référentiels qui sont formalisables, qui évoluent dans le temps, en fonction de l'histoire économique et des attentes de la société.

Depuis quelques mois, à travers les discours des firmes et de leurs organisations représentatives, dans les médias, et dans la société plus généralement, apparaît un regain d'intérêt pour le statut de l'éthique et plus spécialement de l'expertise qu'elle peut apporter. Ses défenseurs la présentent comme facteur de développement et entendent mettre l'économie au service de l'homme, valoriser la cotation des valeurs sociales de l'entreprise, développer les centres de documentation éthique et inventer de nouveaux métiers liés aux enjeux éthiques.

13 En termes de division du travail, l'éthique devient intégrée à la division technique du travail et non plus à la division sociale du travail, telles que-Louis Althusser les a précisées (Althusser. 1995). Face à l'« ancienne » utilisation de l'éthique rimant avec cosmétique (communication symbolique), apparait aujourd'hui une réalité autre, qui lui accorde une place plus profonde et structurante (communication fonctionnelle). L'entreprise du futur souhaite faire de l'éthique une véritable stratégie de développement à long terme et non une simple stratégie de communication. Elle ne sera plus uniquement dévolue au "dircom ", mais agira transversalement dans l'entreprise, concernera tous les services, se manifestera, vivra dans tous les départements, accompagnera tous les processus décisionnels.

14 Parfois simple chargé de mission, selon l'histoire et la nature de l'entreprise dans laquelle le déontologue intervient, sa tâche peut évoluer en fonction des attentes, mais sur le fond, il doit d'abord garantir les principes qui encadrent telle ou telle activité professionnelle réglementée et pouvoir imaginer des procédures originales qui font une place particulière à son intervention. Cet officier de mise en conformité («compliance officer») est présenté comme l'homme qui veille au comportement éthique des personnels dans l'entreprise, au respect de la «morale des affaires». La déontologie devient dans cette perspective moins que la morale, plus modeste, mais aussi plus efficace, plus opérationnelle, et les actions qui en découlent sont censées être cantonnées dans le concret, dans des aspects matériels. Dans la même ligne, apparaissent les « ethics officers ", dont la mission est aussi d'administrer l'éthique dans leur organisation et les programmes de conformité qui s'y rattachent. Ils sont responsables de l'éthique corporative, et maitrisent les instruments utiles pour faire table rase du scepticisme moral à l'intérieur et à l'extérieur de l'entreprise.

La profession est exercée principalement dans les établissements financiers, et pour les entreprises de biens et de services, on appellera aussi le déontologue «responsable éthique de l'entreprise ", souvent très lié à la direction juridique. Il doit accompagner les opérateurs dans leur activité quotidienne, travailler à leur rythme et les "rassurer ", 
dans le souci d'une compréhension systémique de l'organisation, comme le dit Henri Casadesus. "compliance officer» dans l'établissement bancaire J-P. Morgan à Paris (Casadesus, 2000. p.55). Il lui est en effet possible d'influer sur l'ensemble de la structuration de l'entreprise par la manière dont son expertise sera appliquée, puisqu'il n'évalue pas de critères techniques ou financiers, mais intervient sur des questions de gestion des ressources humaines ou de communication. Il ne bénéficie pas d'une autorité scientifique réelle, comme dans les domaines physiques, ou biologiques, où l'avis des experts apparaît souvent incontesté, mais son expertise organisationnelle et ses avis à l'intérieur de l'entreprise ont un très large écho, puisqu'ils touchent aux rapports sociaux.

\section{De réels objectifs managériaux pour l'expert}

16 Les écrits professionnels matériels, techniques, peuvent être pensés comme efficaces, produisant une certaine forme d'organisation bien réelle. Le discours de l'expert sur ces codes assurerait une cohérence à l'intérieur de l'entreprise, autour de l'idée qu'il produit une «logique de formation des identités professionnelles» (Dubar, 2000), et de structuration d'une sphère professionnelle. On retrouve là un effet réel d'amplification des discours éthiques, par un travail stratégique de communication, de relations humaines, qui donne l'occasion aux acteurs de se constituer en groupes professionnels.

17 Le déontologue cherche à établir un lien entre les salariés, entre les salariés et les actionnaires, en leur offrant un nouveau moyen de se connaitre. Cette mise en relation de membres d'une communauté, ces connaissances partagées autour d'éléments discursifs et symboliques, cette perception unifiée du devenir de l'entreprise, des enseignements à tirer de l'éthique, des lignes de conduite, des orientations dans les choix, tout ceci s'inscrit dans les politiques managériales de son entreprise. La question du renouvellement des organisations elles-mêmes est en jeu, et passe par la mobilisation de la subjectivité du salarié, par leur changement, qui joue sur des tensions qui peuvent exister entre logiques d'acteurs et logiques de systèmes (Linhart, 1994).

Danièle Linhart insiste sur ce travail de management à l'intérieur de l'organisation, qui assume de la gestion immatérielle de données, de surveillance, de contrôle, et organise finalement la communication, l'intersubjectivité entre les salariés. Elle voit dans cette démarche "une sous-traitance au salarié lui-même de celte démarche scientifique que préconisait Taylor. de rationalisation et d'organisation du travail, et c'est au salarié de faire l'usage de soi le plus efficace, le plus pertinent possible du point de vue de l'entreprise. » (Linhart. 2000. p. 1). Il revient au salarié de trouver lui-même des solutions aux dysfonctionnements de l'organisation à laquelle il appartient, en se « raccrochant » à ce qui lui est transmis finalement par les experts en termes d'outils de pensée, de valeurs, de culture. La communication servira dans ce contexte à améliorer la productivité et à intégrer les salariés dans les stratégies économiques.

La communication sur l'éthique des affaires, qui relevait surtout d'enjeux identitaires pour l'organisation en quête de valeurs, prend aujourd'hui cette forme d'instrument de contrôle: "elle vise à transformer les salariés en managers ", selon Danièle Linhart (Linhart, 1999, p.3). La communication reliée au discours éthique commence à se substituer, d'une certaine façon, à l'organisation du travail. Plutôt que les formes des codes éthiques, on ne retient que l'évolution des procédures de travail qui est permise. 

phénomènes sociaux peuvent être traités comme des phénomènes naturels. Or, nous savons qu'il est illusoire de posséder une connaissance scientifique sur des ensembles d'individus.

En voulant faire appliquer ces bonnes pratiques, et en imaginant trouver du sens dans son travail, le déontologue va parfois avancer dans le «flou ", sans réussir réellement à analyser les arguments produits par les agents. La légitimité de l'expert, qui est primordiale rappelons-le, passera pourtant par sa connaissance de l'entreprise, sa principale qualité devant être en effet de pouvoir bénéficier d'une approche commune des problèmes. «L'expertise repose sur un capital symbolique dont le contenu a une réalité qui oscille entre la compétence réelle et la réputation » (Floris, 1994. p. 55).

La mission de l'expert rejoint ici une nouvelle fois le domaine de la communication. À défaut de comprendre la réalité de l'organisation dans laquelle il évolue, impossible à maîtriser totalement, son discours prendra nécessairement un caractère impératif et performatif, il devra objectiver et s'auto-institutionnaliser.

Il mettra pour cela à la disposition des salariés, à usage interne, un recueil des dispositions déontologiques, souvent rédigé par ses soins. Il assumera ainsi une mission d'assistance et d'orientation auprès de ses collaborateurs, à partir de documents qui fixent les règles, et que le prestataire et le collaborateur doivent respecter pour se conformer au code de bonne conduite. Un travail de conseil, d'assistance, et d'orientation pour guider ses interlocuteurs et pour appliquer les règles. Il sera même conduit à 
organiser des sessions de formation interne sur ces questions, et s'immergera dans les pratiques professionnelles, avec le souci d'apporter un avis et une réflexion qui éclaireront, favoriseront la circulation de l'information, la participation, la mobilisation et l'implication des salariés. Une mission de pédagogue qui diffuse à tous les échelons de l'entreprise la déontologie, et assumée par la plupart des professionnels (Lignot. 2000. p. 57).

27 A cet égard, les chartes et recueils éthiques s'associent directement à une forme de communication institutionnelle, qui produit un cadre de référence professionnel grâce aux supports que représentent les documents ou les discours touchant à l'éthique d'entreprise. On assiste à l'opérationnalisation du document éthique doublé d'un processus de sensibilisation : "Les outils sont façonnés par l'homme, mais ils façonnent l'homme aussi.» (Bougnoux, 1999. p.1). Ces derniers concourent à la professionnalisation, à la reconnaissance et à la matérialisation de l'activité des déontologues, en leur délimitant des spécificités : production de données, de statistiques, rationalisation de savoir faire par des brochures, des manuscrits, manuels, codes, etc.

C'est bien la formalisation éthique qui permettra de guider les comportements individuels. La «culture implicite» est progressivement remplacée par une "éthique commune et explicite» (Mercier. 1999. p. 17), qui rassure et permet le partage des objectifs. L'expert en gestion symbolique qu'est le déontologue parvient à obtenir des individus un sentiment d'appartenance à l'entreprise et une identification à ses systèmes de valeurs réifiés par des traces écrites (Schwebig, 1982).

Une reconnaissance qui devra aussi passer à l'extérieur de l'organisation, par les salariés eux-mêmes parfois, porteurs de l'image de leur institution, gardien de sa réputation. La diversité des codes de conduite, associée à leur présentation hautement symbolique, servira à rendre lisibles les résultats d'une entreprise donnée, les soi-disant effets concrets de la normalisation qui aura été apportée par l'éthique. Les actionnaires veulent investir dans des entreprises éthiquement correctes, et demandent des explications sur l'origine des produits, les qualifications de ceux qui les ont fabriqués, etc. Leur demande est mise en phase avec la vision et les objectifs du dirigeant - et donc du déontologue qui devient progressivement, si la communication est réussie, la vision et les objectifs partagés par le corps social tout entier.

Ces éléments décrits représentent-ils une nouveauté ? La fonction éthique relevait auparavant du Directeur des Ressources Humaines, dont les tâches comportent aussi cette connotation humaniste et transversale. Où se trouve le renouveau dans ces pratiques associées au « déontologue »?

\section{L'expert déontologue : Recomposition d'ordre matériel ou production immatérielle et qualitative pour une pérennisation de l'organisation?}

Notre hypothèse est de comprendre cette volonté de matérialiser l'éthique par une profession comme une tentative pour réinventer une forme de médiatisation des entreprises, en leur sein, qui se bornerait à une simple pétition de principes vertueux sans conséquences pratiques véritables. 

l'emporte, et s'impose même, ne laissant finalement place qu'à de nouvelles manières de communiquer pour les managers, à l'intérieur de l'organisation ou dans l'espace public.

\section{L'expertise éthique comme vecteur symbolique supplémentaire de validation des règles}

Les codes éthiques sont faits par et pour l'organisation elle-même, et leur idée fait corps avec la personne du déontologue. Ils accompagnent aujourd'hui les réflexions sur l'action, et ne sont plus l'apanage des juristes, mais il semble que l'expertise qu'ils permettent n'apporte pas autre chose qu'une dimension qualitative forte pour l'organisation.

La forme de communication, non palpable, «du sens et du travail » (Floris, 1994. p. 62), qui était celle des dircoms il y a dix ans, peut aisément se rapprocher aujourd'hui de la mission dévolue aux déontologues. À la manière des directeurs de communication qui formalisaient la communication dans le sens d'une réduction du sens du travail au bénéfice de sa productivité, le déontologue rationalise les subjectivités, dissémine un discours autour de la notion de " consommateur citoyen ", il produit une culture éthique.

On note ainsi des tentatives de changement par rapport aux pratiques sociales, organisationnelles et matérielles préexistantes. «La question du changement est devenue transversale à un ensemble de discours, notamment en psychologie, sociologie, sciences de gestion, sciences de l'information et de la communication. » (Bernard. 2000. p).

Autour de la question de l'expertise éthique et de son applicabilité à l'intérieur de l'entreprise, on assiste à une tentative visant à définir justement des logiques d'action nouvelles, en lui accordant des ramifications plus grandes à l'intérieur des organisations, des missions précises, en la faisant intérioriser par les salariés. Ses valeurs transparence, loyauté, exemplarité - ne sont pas nouvelles, mais ce qui est nouveau, c'est leur affichage, leur haute priorité pour l'entreprise tout entière, qui aspire au consensus, à la coopération, au partenariat. "Qui peut être contre la qualité des biens et services offerts?", interrogeait Jean-Pierre Durand (Durand, 2000. p. 22).

Nous nous trouvons là face à des stratégies de management assez inédites, et Michel Le Net, Président du Cercle d'Ethique des Affaires, rappelle qu'une étude menée par l'Observatoire éthique des entreprises - dépendant du C.E.A - (Le Net. 2000. p. 105) a montré qu'aujourd'hui en effet, l'éthique est devenue un outil de management indispensable à la pérennité des organisations, qu'elle imprègne tous les rouages de l'entreprise.

Les effets de sa présence ne nous semblent que communicationnels. L'étymologie du mot déontologie signifie d'ailleurs « discours sur ce qu'il faut faire » (Médina, 2000, p. 27), sur ce que sont les règles, règles de comportement en société, comme les règles professionnelles à suivre à l'intérieur de l'entreprise, fixées par les institutions ou les ordres professionnels, nous rappelle Yves Médina, qui œuvre pour le cabinet d'audit Price Waterhouse Coopers. Son rôle revient peut-être surtout à définir les relations des acteurs à l'intérieur de l'entreprise. Les documents éthiques ont toujours constitué un lieu de mémoire pour les règles. La réflexion en la matière est normative, et elle s'enracine aussi dans les émotions, l'identification à autrui, l'immatériel.

Le déontologue remplirait ce rôle d'interface permettant la circulation matérielle, technique des idées et des valeurs, porteur de la bonne parole. Derrière la matérialité des 
dispositifs de chartes éthiques, on s'aperçoit qu'ils servent encore essentiellement à penser, connaître, faire croire, pour transmettre, inculquer, et avoir en commun des représentations, des règles. «En ce qui concerne les rapports d'expertise ou d'études, nous soulignerons uniquement le fait qu'ils sont ensuite, comme toute information à contenu stratégique, contextualisés dans les dynamiques de pouvoir interne, ce qui peut conduire parfois tout simplement à les déposer dans un tiroir sans les exploiter.» (Bernard, 1998, p. 52). Il est nécessaire en effet de voir en aval de ces interventions et opérations d'interface, les formes d'appropriation des savoirs par les acteurs.

C'est la culture qui permet la transmission, impulsée par le déontologue qui contribue à l'élaboration de règles dans l'entreprise, à les faire partager par les différentes parties prenantes : personnels, clients actionnaires. Son rôle d'acculturation et de structuration est important, et va jusqu'à mettre en œuvre des contrôles d'application.

41 «En fait, les exigences productives nées de la compétence (qualité, variété, productivité, etc.) qui apparaissent comme nouvelles, sont aussi des créations et des inventions destinées à justifier l'obligation, pour les salariés, de s'engager sur les objectifs de l'entreprise, de se mobiliser et de faire preuve de loyauté par rapport à celle-ci.» (Durand. 2000, p. 22). Cela pose la question de la convergence d'intérêts entre salariés et hiérarchie, autour des valeurs éthiques professionnelles (Zarifian, 1999, p. 141).

\section{L'immatérialité de la " gestion grise » par l'expertise : la question des effets du travail du déontologue?}

L'éthique autrement proposée par les directions d'entreprises accorde au déontologue ce statut si particulier de celui qui ne doit pas prendre position, selon un principe de neutralité bienveillante. On évoquera parfois la notion de « gestion grise » (Besset (du) et Oriol, p. 11).

Autrement dit, le déontologue, n'aura pas d'autorité hiérarchique, mais une autorité de compétence, une autorité morale, et en tant qu'expert, il tâchera d'adopter une posture de tiers impartial qui est spécifique à la gestion des rapports des individus dans l'organisation, plus qu'à des problèmes techniques ou matériels. Selon les textes de la Commission des Marchés Financiers, qui gère la profession de déontologue dans les milieux financiers, celui-ci doit réussir à créer des cloisons étanches entre les diverses entités du groupe dans lequel il exerce son activité, ce que le jargon décrit comme des « murailles de Chine » (CMF, 1994), qui permettent de voir d'un côté et de l'autre du mur, pour éviter les conflits. Il rendra bien évidemment compte à l'organe exécutif ou au directoire, qui lui accordent leur confiance, de ses observations de terrain. Aux décideurs ensuite de prendre les décisions importantes, et le déontologue n'aura t'ait qu'instruire les dossiers, en travaillant à se séparer du message, à articuler discrètement, sous la surveillance de sa hiérarchie, la pensée de l'ingénierie sociale à celle de l'ingénierie symbolique.

Son travail suppose qu'il y ait une production symbolique à l'appui, de l'écoute, un dialogue constructif de « l'humilité et [de la] modestie car l'éthique n'est pas blanche ou noire, elle est souvent grise. Il s'agit de retenir le meilleur compromis possible dans l'intérêt du plus grand nombre. » (Besset (du) et Oriol, p. 13). Dans la société, certains s'élèvent pour accuser l'éthique dans le domaine des nouvelles biotechnologies, d'impuissance, d'être «le bureau des relations publiques » (Pol-Droit. 2000). Ces griefs 
pourraient aisément se transférer à la sphère de l'entreprise. Roger Pol-Droit taxe l'éthique d'immobilisme, et précise que le débat en la matière agit comme " dernier appel des principes avant l'arrivée des bons de commande» (Pol-Droit. 2000). L'éthique se donne dans ce contexte pour rôle de ralentir, d'arrêter l'accélération d'un mouvement ignorant les conséquences et le sens de ses actes.

Nadine Fresco d'ajouter que l'éthique n'est qu'un «jardin d'acclimatation », servant à habituer les esprits aux mutations en cours (Fresco, 2000). En effet, l'expert déontologue se limite à évaluer le fonctionnement de l'organisation, sa qualité, et pas vraiment les performances elles-mêmes, sur lesquelles il n'a aucune prise réelle. L'expert doit rester neutre, en retrait, et lors de ses audits ou conseils, il devra veiller au respect du secret professionnel, à son indépendance, etc. Il ne peut être seul dans l'organisation, et compte à la fois sur la hiérarchie, car les réticences sont fortes, et en même temps, sur le soutien des opérateurs, sinon, il ne peut œuvrer efficacement.

En cela, son intervention est globale, encore une fois fondée sur le compromis, il doit la mener de façon matricielle, transversale, sur les structures verticalisées d'un réseau, afin d'apporter son point de vue, d'alerter au travers d'échanges, et d'aider à entretenir une "tension déontologique», comme l'écrit Yves Médina (Médina, 2000, p.31). Il contribuera, en se plaçant au dessus des débats, au développement harmonieux à la firme dans laquelle il exerce ses compétences, sans que sa mission d'orientation et d'assistance qui guide les uns et les autres ne soit trop perceptible. Il instille l'« esprit » des règles (Karlin. 1998. p. 48), établit des ponts entre les directions, les responsables opérationnels et les diverses parties prenantes, en leur expliquant « leur raison d'être » (De Presle, 2000, p. 46).

\section{Mystification du statut de l'expertise éthique}

47 Le support que représente le code éthique finit par être caché par le message du déontologue; le récepteur aveuglé par les conditions d'acheminement du message, fasciné par la performance symbolique et culturelle de l'expert.

Toutes sortes de projets d'innovation technique ou sociale sont associés à des raisons dites éthiques. Quand on y regarde d'un peu plus près, on s'aperçoit, que, parmi ces raisons dites éthiques, un certain nombre devraient probablement être qualifiées autrement. Il serait plus juste, d'après Ruwen Ogien, de dire qu'il s'agit de « raisons psychologiques, sociales, politiques, juridiques, techniques, etc.(...)» (Ogien, 2000).

Ce genre de confusion ne doit pas nous surprendre. Il n'est pas très facile de distinguer les raisons éthiques de celles qui ne le sont pas. Il vaut mieux, dans ce domaine, adopter une stratégie minimaliste ou déflationniste, en réservant la qualification d'éthiques à un groupe très limité de raisons. C'est ainsi que certains formulent des jugements pessimistes à propos de l'avenir de l'éthique. D'après Ruwen ogien toujours, le débat éthique tel qu'il se déroule actuellement est une mystification. A première vue, l'éthique penche du côté de la prudence, de la précaution, de l'interdiction. C'est, en quelque sorte, une barrière, une force conservatrice, surtout dans le domaine des organisations marchandes ou non marchandes.

En réalité, le débat éthique contribue à désarmer les résistances aux innovations aussi inquiétantes soient-elles, ce qui arrange bien les intérêts corporatistes (médecins) ou ceux des entreprises. Lorsque cette «fonction cachée " deviendra manifeste, plus 
personne ne prendra l'éthique au sérieux. Ce sera la fin de l'éthique ou, au moins, la fin du débat éthique tel que nous le connaissons actuellement (avec ses comités, sa littérature à succès, etc.).

\section{Conclusion}

51 La création d'une fonction de déontologue en France est-elle une tendance lourde ou un épiphénomène de mode ? A vrai dire, il existe peu d'informations à ce jour sur l'influence des documents éthiques dans les comportements quotidiens des individus dans les entreprises. Cependant, il est permis de supposer que leur présence peut prendre une dimension symbolique voire managériale forte. La formalisation éthique est saluée par certains non pas comme l'avènement d'une nouvelle profession mais plutôt comme un détournement, une perversion de la réflexion éthique, comme "un abus de pouvoir » (Obrecht, 1994, p. 45) car elle entraîne un déplacement de la frontière traditionnelle entre la vie privée des individus et leur vie professionnelle.

Acheter éthique, parler éthique, placer éthique, gouverner éthique... Etre éthique ou ne pas être, c'est l'injonction contemporaine que nous avons tenté de décrire à travers la question de la matérialité de l'éthique dans les entreprises. Elle rejoint une autre réflexion plus globale, celle de la rationalité, de la légitimité et de la pérennité des modes de gouvernance qui s'imposent aujourd'hui dans les entreprises en se préoccupant du seul intérêt des actionnaires. Quel type de confiance les salariés peuvent-ils accorder à ce type de système? Quelle continuité, quelles règles du jeu concrètes peuvent-ils $\mathrm{y}$ déceler ? Le problème pour les entreprises aujourd'hui est de refonder leur légitimité aux yeux de leurs salariés, et la communication institutionnelle, immatérielle, devrait confirmer son développement dans les prochaines années.

\section{BIBLIOGRAPHIE}

ALTHUSSER. L. Sur la reproduction. Paris : PUF/Actuel Marx. 1995. p. 58-60.

BERNARD, F., Communication organisationnelle et usage des Sciences Humaines, in LE MOËNNE, C. (texte réunis par), Communications d'entreprises et d'organisations. Rennes : PUR, 1998, pp. 49-78.

BERNARD, F., Le débat individu/organisation/communication - Filiations, itérations et évolutions, in Sciences de la société. n 50-51, Toulouse : Lerass. 2000, pp. 20-30.

BESSET (du). P. et ORIOL, F., Déontologue : un nouveau métier in Entreprise éthique, $\mathrm{n}^{\circ} 12$, Bruxelles : De Boeck Université, avril 2000. pp. 11-15.

BOUGNOUX, D., Sur la médiologie : conférence. Rennes, mars 1999. CANTO-SPERBER. M., « ... ». Revue Esprit, mai 2000. CASADESUS, H., LIGNOT, J-P., Etre déontologue dans une banque française et dans une banque américaine : le cas de la Société Générale et de JP Morgan, in Entreprise éthique, $\mathrm{n}^{\circ}$ 12, Bruxelles : De Boeck Université, avril 2000, pp. 53-60. 
CHAPOULIE, J-M., Sur l'analyse sociologique des groupes professionnels. Revue française de sociologie, $\mathrm{n}^{\circ} 14$, Paris, 1973, pp. 86-114. DUBAR, C, La socialisation, construction des identités sociales et professionnelles, Paris : Armand Colin, 2000.

DURAND, J-P., Les enjeux de la logique de compétence, in Annales des mines, décembre 2000, pp. 16-28.

ELIET, G., Le déontologue des établissements financiers : l'avènement d'un nouveau métier, in Entreprise éthique, $\mathrm{n}^{\circ}$ 12, Bruxelles : De Boeck Université, avril 2000, pp. 33-40.

FLORIS, B., Les ingénieurs du sens, in Sciences de la société, n 32. Toulouse : Lerass, 1994, pp. 53-69.

FRESCO, N. Critique de la raison éthique, Paris, Libération. 16 décembre 2000.

HOTIER, H. (textes recueillis par), Ethique et communication, Bordeaux, GREC/O. ISIC. 1990.

KARLIN, M., Les règles de bonne conduite sur les marchés financiers, in Revue Banque, $\mathrm{n}^{\circ} 598$, décembre 1998, pp. 48 et $\mathrm{s}$.

LE NET, M., L'éthique : condition de la pérennité des organisations, in Entreprise éthique, $\mathrm{n}^{\circ} 12$. Bruxelles : De Boeck Université, avril 2000. pp. 105-111.

LINHART, D., La modernisation des entreprises. Paris : La découverte, coll. Repères. 1994.

LINHART, D., Le discours d'accompagnement des TIC dans les entreprises, La communication entre libéralisme et démocratie, Paris. Colloque. 12/12/2000.

MAUSS (revue du). Ethique et économie - L'impossible remariage Paris : La Découverte/Mauss, 2000.

MEDINA, Y., Le déontologue n'est pas un moraliste, in Entreprise éthique. $\mathrm{n}^{\circ} 12$, Bruxelles : De Boeck Université, avril 2000, pp. 25-31.

MERCIER. S. L'éthique dans les entreprises éditions, Paris : La Découverte \& Syros, coll repères, 1999. OBRECHT, J-J., L'éthique et le nouvel entrepreneur, in Gestion 2000. 1994. OGIEN. R., Critique de la raison éthique, in Libération, 16 décembre 2000. POL-DROIT, R., in La Croix, 13 novembre 2000.

PRESLE (de la), Déontologue, responsable éthique : apparence ou réalité ?, in Entreprise éthique, $\mathrm{n}$ -12. Bruxelles : De Boeck Université, avril 2000. pp. 33-40.

SCHWEBIG, P., Les communications de l'entreprise, Paris : Mac Graw Hill, 1982.

TREPOS, J-Y., La sociologie de l'expertise, Paris : PUF, 1996. ZARIFIAN, P., Objectif compétence - Pour une nouvelle logique. Paris : Editions Liaisons, 1999.

\section{Presse professionnelle}

Conseil des Marchés Financiers, Règlement général article 31 4. 2000.

\section{RÉSUMÉS}

Aujourd'hui, la stratégie affichée des, directions d'entreprises consiste à vouloir intégrer l'éthique à des objectifs matériels, touchant au contrôle et à la recomposition des organisations (nouveaux métiers, nouvelles fonctionnalités). On se situe là dans les grandes entreprises d'origine plutôt anglo-saxonne qui possèdent des filiales en France et en Europe, et qui sont les plus touchées par l'apparition des nouveaux métiers de "déontologues", dont la mission consiste à jouer un rôle d'experts (90 \% des grandes entreprises américaines ont une politique éthique formalisée (Mercier, p. 10. 1999). Nous souhaitons faire le point sur les motivations des dirigeants d'entreprise du XXI ${ }^{\mathrm{e}}$ siècle : les effets de matérialité de l'éthique qu'ils proclament par 
leurs discours sont bien réels, mais nous formulons l'hypothèse que la mission du déontologue a essentiellement pour objet d'innover en matière de stratégies de communication, afin d'orienter celles-ci vers une démarche de " gestion du risque de réputation ».

Today the strategy overtly shown by enterprise managements consists in meaning to integrate ethics into material objectives, related with controlling and recombining organisations (new jobs, new functionalities). We are talking here of large enterprises, rather of Anglo-Saxon origin, that own susidiaries in France and in Europe, and are more touched by the emergence of new ideontologicaly personnels, mission it is to intervene as experts (90\% of major American enterprises have a formalised ethical policy (Mercier. p. 10. 1999). We would like to take stock of the motivations of enterprise managers in the $21^{\text {st }}$ century: the effects of materialisation of ethics that they proclaim in their speeches are indeed real, hut we formulate the hypothesis that the mission of the deontologist is essentially to innovate in matter of communicating strategies, in order to turn them into a « management of the reputation risk » attitude.

INDEX

Mots-clés : déontologie, éthique, gouvernance, production qualitative immatérielle

\section{AUTEUR}

\section{CATHERINE LONEUX}

Catherine Loneux est Maître de Conférences à l'Université de Rennes 2, membre du CERCOR, membre du Groupe d'Études et de Recherches sur les Communications organisationnelles (Org \& Co) de la Société Française des Sciences de l'Information et de la Communication (SFSIC) et Vice-Présidente de la Commission « International » de la SFSIC. 